\title{
Physiological factors associated with low bone mineral density in female endurance runners
}

\author{
M Burrows, A M Nevill, S Bird, D Simpson
}

Br J Sports Med 2003;37:67-71

\begin{abstract}
Objective: To explore potential factors that could be associated with low bone mineral density (BMD) in female endurance runners.

Methods: Fifty two female endurance runners (1500 m to marathon), aged 18-44 years, took part. Body fat percentage, lumbar spine BMD, and femoral neck BMD were measured using the Hologic QDR 4500w bone densitometer. Data on training, menstrual cycle status, osteoporosis, and health related factors were obtained by questionnaire. Dietary variables were assessed from a prospective seven day dietary record of macronutrients and micronutrients.

Results: The mean (SD) lumbar spine and femoral neck BMD were $1.11(0.11)$ and $0.89(0.12) \mathrm{g} / \mathrm{cm}^{2}$ respectively. A backward elimination regression analysis showed that age, body mass, body fat, distance run, magnesium, and zinc intake were the variables significantly associated with BMD. Lumbar spine $B M D\left(\mathrm{~g} / \mathrm{cm}^{2}\right)=-1.90+(0.0486 \times$ age $($ years $))+(0.342 \times \log$ mass $(\mathrm{kg}))-\left(0.000861 \times\right.$ age $^{2}$ (years) $)-\left(0.00128 \times\right.$ distance $\left(\mathrm{km} /\right.$ week)), with an $R^{2}=30.1 \%$ (SEE $=0.089(95 \%$ confidence interval (Cl) 0.05 to 0.23); $p<0.001)$. Femoral neck $B M D\left(g / \mathrm{cm}^{2}\right)=-2.51-(0.00989 \times$ age (years) + $(0.720 \times \log$ mass $(\mathrm{kg}))+(0.000951 \times$ magnesium $(\mathrm{mg} /$ day $))-(0.0289 \times$ zinc $(\mathrm{mg} /$ day $))-(0.00821$ $\times$ body fat $(\%))-\left(0.00226 \times\right.$ distance $(\mathrm{km} /$ week) $)$, with an $R^{2}=50.2 \%($ SEE $=0.100(95 \% \mathrm{Cl} 0.06$ to 0.22$) ; p<0.001)$. The negative association between skeletal BMD and distance run suggested that participants who ran longer distances had a lower BMD of the lumbar spine and femoral neck. Further, the results indicated a positive association between body mass and BMD, and a negative association between body fat and BMD.

Conclusions: The results suggest a negative association between endurance running distance and lumbar spine and femoral neck BMD, with a positive association between body mass and femoral neck and lumbar spine BMD. However, longitudinal studies are required to assess directly the effect of endurance running and body mass on $B M D$, and to see if the addition of alternative exercise that would increase lean body mass would have a positive effect on BMD and therefore help to prevent osteoporosis.
\end{abstract}

See end of article for
authors' affiliations

Correspondence to: Dr Burrows, University Health and Biosciences Stratford Campus, Romford Road, London

E15 4LZ, UK

m.burrows@uel.ac.uk

Accepted 7 May 2002 
0-9 cycle a year; amenorrhoeic $=0-3$ cycles a year; eumenorrhoeic $\geqslant 10$ cycles a year). The questionnaires collected information on the history of the menstrual, training, and dietary status, as well as any recent changes (within the last year). A prospective, seven day dietary record was also completed, from which average energy (MJ/day), zinc (mg/day), magnesium (mg/day), phosphorus ( $\mathrm{mg} /$ day), and calcium ( $\mathrm{mg} /$ day) intake over this period was assessed (Dietmaster, version 4.0; Swift Computers Ltd, Phoenix, Arizona, USA).

Participants were weighed (wearing minimal clothing) to the nearest $0.1 \mathrm{~kg}$ (Balance Beam Scale; Seca, Germany), and height recorded to the nearest centimetre (stadiometer attached to Balance Beam Scale). Bone densitometry was used to assess body fat percentage and BMD. BMD was measured at the lumbar spine (L2-L4) and the femoral neck, and body fat was assessed during a whole body scan, using dual energy $x$ ray absorptiometry (Hologic, QDR 4500w, Hologic Inc, Waltham, Massachusetts, USA). The standard Hologic protocol for positioning the lumbar spine and femoral neck was used. Bone mineral results were expressed as BMD $\left(\mathrm{g} / \mathrm{cm}^{2}\right)$. All scans were conducted and analysed in the osteoporosis unit at the Kent and Canterbury Hospital. The in vivo and in vitro precision of dual energy $x$ ray absorptiometry for BMD in the laboratory was less than $1 \%$ for both the lumbar spine and femoral neck.

Statistical analysis was carried out using the Minitab statistical package (version 12.1). A backward, elimination log-linear regression model was used beginning with a saturated model incorporating all possible covariates and then sequentially eliminating the least important variables (based on the size of the $t$ statistic), to assess the impact of all the variables on bone density at the femoral neck and lumbar spine sites. On the basis of proportional models proposed by Nevill and Holder, ${ }^{13}$ the following multiplicative model was proposed for BMD of the lumbar spine (L2-L4) and femoral neck:

$B M D=$ weight $^{k} \times \exp \left(c+(d \times\right.$ age $\left.)+\left(e \times \operatorname{age}^{2}\right)\right) \times \epsilon$

The model can be linearised with a log transformation:

$\log _{e}(B M D)=k \times \log _{e}($ weight $)+c+(d \times$ age $)+(e \times \text { age })^{2}+\epsilon_{1}$

and, provided that the error term $\epsilon_{1}$ has a normal distribution, linear regression can be used to estimate the parameters $\mathrm{k}, \mathrm{c}$, $\mathrm{d}$, and e. Note that the proportional error ratio term in equation ( 1 ) is dimensionless-that is, a percentage error. Clearly, there is some operational advantage to incorporating age and age $^{2}$ within an exponential term in equation (1), as well as providing a more plausible asymptotic decline for BMD with

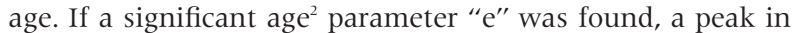
BMD had been identified, suggesting that BMD rises and peaks at age $=-\mathrm{d} / 2 \mathrm{e}$ (using elementary differential calculus) and subsequently declines. The model can be developed to incorporate other factors of interest (within the exponential term) such as body fat, dietary macronutrients and micronutrients, and the distance walked a week $(\mathrm{km})$.

To assess for the assumption of normality, an AndersonDarling normality test and a Box-Cox transformation were used. Box and $\operatorname{Cox}^{14}$ suggested transforming the dependent variable $(\mathrm{Y})$ using either $\mathrm{Y}^{\prime}=\left(\mathrm{Y}^{\lambda}-1\right) / \lambda$ if $\lambda=1$ or $\mathrm{Y}^{\prime}=\log (\mathrm{Y})$ if $\lambda=0$, the value of the parameter $\lambda$ being chosen to maximise the log-likelihood function. The transformation then provides residuals that best approximate to a normal distribution with constant variance. In the case of BMD of the lumbar spine (L2-L4) and the femoral neck, the optimal transformation parameters were $\lambda=0.09$ and $\lambda=0.13$ respectively, which were not significantly different from $\lambda=0$, confirming that a log transformation of BMD was near optimal.
Table 1 Summary data for all variables $(n=52)$

\begin{tabular}{ll}
\hline & Female runners \\
\hline Age (years) & $31(5)$ \\
Height (cm) & $165(6)$ \\
Body mass (kg) & $57.3(6)$ \\
Body fat (\%) & $20(6)$ \\
Age at onset of training (years) & $23(8)$ \\
Distance run (km/week) & $51(28)$ \\
Training per week (hours) & $8(5)$ \\
Years training & $8(6)$ \\
Sessions per week & $5(2)$ \\
Time off training (months) & $3(5)$ \\
Menarcheal age (years) & $14(2)$ \\
Gynaecological age (years) & $17(6)$ \\
Cycle frequency/year & $11(2)$ \\
Cycle flow (days) & $5(1)$ \\
OCA (months) & $57(54)$ \\
Average energy intake (M)/day) & $7.5(2)$ \\
Calcium (mg/day) & $831(257)$ \\
Magnesium $(\mathrm{mg} /$ day) & $257(61)$ \\
Zinc (mg/day) & $7.5(0.7)$ \\
Phosphorus (mg/day) & $1166(260)$ \\
Spinal BMD (g/cm²) & $1.12(0.1)$ \\
Femoral neck BMD (g/ $\left./ \mathrm{cm}^{2}\right)$ & $0.9(0.1)$ \\
\hline
\end{tabular}

Values are mean (SD). Gynaecological age is years since menarche; cycle flow is average length of menstrual flow; Cycle frequency/year is average menstrual cycles experienced each year.

$O C A$, Length of oral contraceptive use in months; BMD, bone mineral density.

BMD values were compared with the Hologic normal range for the lumbar spine $\mathrm{e}^{15}$ and the National Health and Nutritional Examination Survey ${ }^{16}$ for the femoral neck.

Pearson's product-moment correlations were performed between dependent and independent variables to assess the relations.

Alpha was set at 0.05 for all statistical procedures.

\section{RESULTS}

Table l summarises the participant characteristics for all variables.

Of the 52 participants, the menstrual questionnaire classified $12(23 \%)$ as oligo/amenorrhoeic (only one participant was amenorrhoeic), and $40(77 \%)$ as eumenorrhoeic. Further, 46 (88\%) had or were currently using various forms of oral contraceptives over a period of 59 (54) months. The daily energy intake for all participants over the seven day period was 7.5 (2) MJ/day, which placed them below the reference nutrient intake for some vitamins and minerals (table 1). There was no significant correlation $(r=0.04)$ between energy intake and distance run per week.

The mean (SEM) BMD for all participants at the lumbar spine and femoral neck were $1.11(0.11)$ and $0.89(0.12) \mathrm{g} / \mathrm{cm}^{2}$ respectively. The BMD values for the lumbar spine and femoral neck were on average -0.43 and +0.45 SD from those of age matched participants in the databases. Of the cohort, two and one of the participants were classified as osteoporotic at the lumbar spine and femoral neck respectively. Osteopenia was diagnosed in nine and six of the participants at the lumbar spine and femoral neck respectively.

The backward elimination log-linear regression analysis showed that log lumbar spine BMD $\left(\mathrm{g} / \mathrm{cm}^{2}\right)$ was significantly associated with age (years) $(p=0.032), \operatorname{age}^{2}(p=0.021), \log$ body mass $(\mathrm{kg})(\mathrm{p}=0.007)$, and distance run $(\mathrm{km} /$ week $)(\mathrm{p}=$ $0.035)$. It was calculated that lumbar spine BMD increased up to the age of $(-\mathrm{d}) /(2 \mathrm{e})=0.0486 /(2 \times 0.000861)=28.2$ years, where it peaked and declined thereafter (parameters taken from the fitted regression model). The lumbar spine BMD was associated with body mass only, not body fat percentage, suggesting that it may be the lean component of body mass that 


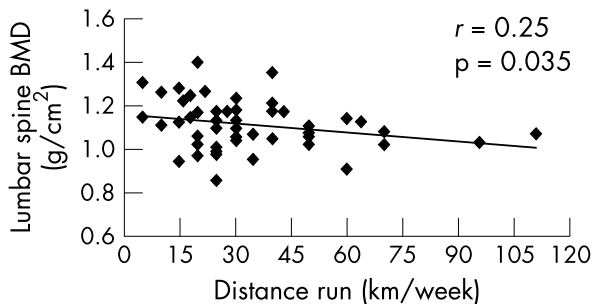

Figure 1 Relation between distance run a week and bone mineral density (BMD) at the lumbar spine.
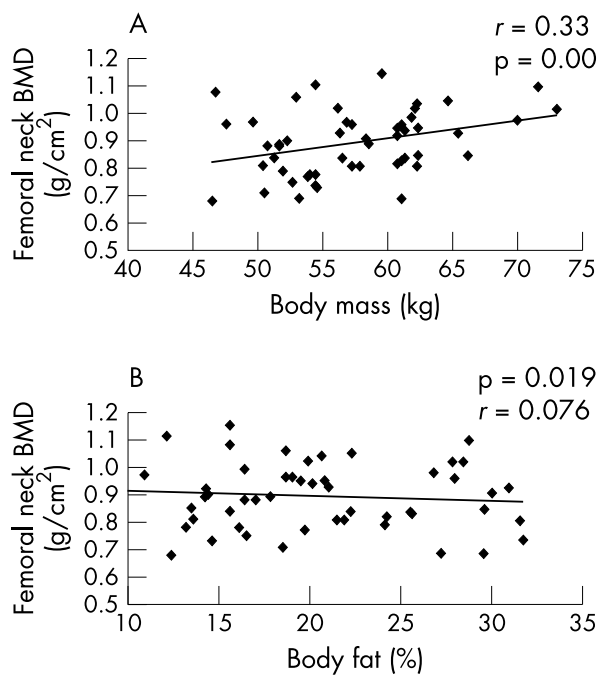

Figure 2 Relation between body composition and femoral neck bone mineral density (BMD). (A) BMD and body mass; (B) BMD and body fat (\%).

is associated with lumbar spine BMD. Distance run was negatively associated with lumbar spine BMD, with participants who ran the longer distances appearing to have a lower BMD (lower by $1 \%$ in participants running a further $10 \mathrm{~km} /$ week) (fig l).

Log transformed femoral neck BMD was significantly associated with distance $(\mathrm{km} /$ week) $(\mathrm{p}=0.006)$, age (years) $(\mathrm{p}=$ $0.002), \log$ body mass $(\mathrm{kg})(\mathrm{p}=0.000)$, magnesium intake $(\mathrm{mg} /$ day) $(\mathrm{p}=0.006)$, zinc intake $(\mathrm{mg} /$ day $)(\mathrm{p}=0.009)$, and body fat percentage $(p=0.019)$. The regression model indicated that magnesium intake $(\mathrm{mg} /$ day) was positively related, whereas zinc intake (mg/day) was negatively related to femoral neck BMD. Distance run ( $\mathrm{km} /$ week) was negatively associated with femoral neck BMD, with participants who ran the longer distances appearing to have a lower BMD (lower by $2 \%$ in participants running a further $10 \mathrm{~km} /$ week). A significant age related decline in femoral neck BMD was observed but the quadratic age ${ }^{2}$ parameter was not significant, suggesting that a peak in femoral neck BMD might have occurred in late adolescence (the teenage years) rather than early adulthood. Once again, body mass was positively associated with BMD, whereas body fat percentage was negatively associated with $\mathrm{BMD}$, suggesting that it may be the lean component of body mass that was positively associated with BMD at this site (fig 2).

The resulting regression equations were:

log lumbar spine BMD $\left(\mathrm{g} / \mathrm{cm}^{2}\right)=-1.90+(0.0486 \times$ age (years) $)+(0.342 \times \log$ mass $(\mathrm{kg}))-\left(0.000861 \times\right.$ age $^{2}$ (years) $)-(0.00128 \times$ distance $(\mathrm{km} /$ week $))$

$R^{2}=30.1 \%$; SEE $=0.089$ (95\% confidence interval (CI) 0.05 to $0.23) ; \mathrm{p}=0.002$ log femoral neck BMD $\left(\mathrm{g} / \mathrm{cm}^{2}\right)=-2.51-(0.00989 \times$ age (years $))+(0.720 \times \log$ mass $(\mathrm{kg}))+(0.000951 \times$ magnesium $(\mathrm{mg} /$ day $))-(0.0289 \times$ zinc $(\mathrm{mg} /$ day $))-$ $(0.00821 \times$ body fat $(\%))-(0.00226 \times$ distance $(\mathrm{km} /$ week $))$

$R^{2}=50.2 \%$; $\mathrm{SEE}=0.100(95 \%$ CI 0.06 to 0.22$) ; \mathrm{p}<0.001$.

Note that by taking antilogs of the SEEs for lumbar spine and femoral neck BMD regression models, we obtained percentage errors of $9.3 \%$ (95\% CI 5.1 to $25.8 \%$ ) and $10.5 \%$ ( $95 \%$ CI 6.1 to $24.6 \%$ ) respectively.

\section{DISCUSSION}

It has been thought that physical activity may help to offset the bone loss in female runners with inadequate diets, ${ }^{17}$ and it has been shown to exert a positive effect on BMD in postmenopausal women. ${ }^{15}$ As the frequency, duration, and intensity of the training regimens of endurance athletes far exceed the exercise of most postmenopausal women, ${ }^{15}$ one may expect the activity to exert a protective effect against bone loss in such athletes. However in this study, after controlling for differences in body size and age, distance run per week was negatively associated with lumbar spine and femoral neck BMD. The regression analysis indicated that participants who ran longer distances had a lower femoral neck and lumbar spine BMD (refer to the negative beta weights in the regression equations; $\mathrm{p}<0.05$ ) than those who ran shorter distances. Indeed, the association suggested that participants who ran $10 \mathrm{~km} /$ week further than other participants had a $1 \%$ lower lumbar spine BMD and a 2\% lower femoral neck BMD, regardless of the age of training onset. As there is evidence to suggest that low BMD is associated with increased fracture risk, ${ }^{18}$ such runners may be at an increased risk of fracture. However, these results should be interpreted with caution because the study was cross sectional and does not show a cause and effect relation. Further longitudinal studies are therefore required to support such claims.

Research has also suggested that long distance runners may have more dietary deficiencies, ${ }^{1}{ }^{19}$ and that this may affect BMD more than the training per se. However, none of the dietary variables measured here were significantly associated with lumbar spine BMD. This agrees with previous findings, ${ }^{20}$ which suggests that only abnormal and restrictive eating behaviours are related to low BMD and future fracture risk. However, other studies ${ }^{10}{ }^{19}$ have suggested that low energy intake can alter luteinising hormone pulsatile activity and decrease BMD levels. Such a mechanism may be site specific, for, although we did not find any direct relation between energy intake and BMD at any site, femoral neck BMD was significantly associated with magnesium and zinc intake, with higher magnesium and lower zinc intakes related to higher femoral neck BMD values. However, the low magnesium and zinc intakes recorded may reflect an undernourished state of the runners (7.5 (2) MJ/day while running 51 (28) km/week) and thus the effect of a low energy intake on BMD. ${ }^{3}$ Indeed, the dietary intakes were measured by a seven day prospective dietary intake record which relies heavily on the honesty and accuracy of the participants reporting, so the results need to be interpreted with some caution until further studies are completed. However, if the micronutrients are looked at individually, low levels of magnesium could downregulate parathyroid hormone secretion, decreasing calcium uptake and altering the bone remodelling process. ${ }^{21}$ However, the role of zinc in regulating BMD is less clear. Zinc was previously thought to exert a positive influence on BMD and to be essential for bone metabolism. ${ }^{21}$ Thus the negative influence of zinc on femoral neck BMD in this study was surprising. One possible clue may come from the way zinc affects the immune system by increasing osteoclastic activity. ${ }^{21}$ 
Another aspect to consider is the actual forces applied to the limbs during running, as previous research has suggested that they are crucial to BMD. ${ }^{22}$ Athletes involved in sports and training where forces applied to limbs are in excess of 10 times body weight (gymnastics, weightlifting, and volleyball) have been found to have higher BMD than those involved in sports where forces are only in the range of 5-10 times body weight, such as endurance running. ${ }^{24}$ It seems that, although running includes many more cycles of foot strike and thus force application than these other sports, the lower loads are not as stimulatory to bone accretion. This may explain our findings that long running distances appear to be associated with low BMD, because the longer distances covered would be associated with lower intensity exercise and thus lower forces being applied to the limbs. This supports the theory of Frost, ${ }^{22}$ in which a minimum force is required to elicit a bone remodelling response, and forces above this threshold would stimulate modelling at bone sites, resulting in a net bone gain. Therefore, magnitude of loading to the bone may be more important than the number of cycles, repetitions, or exercise mode. ${ }^{25} 26$

One would therefore expect body mass to have a positive effect on BMD. Indeed, this study suggests that body mass has a positive association with lumbar spine and femoral neck BMD. However, the regression analysis indicates that it is body mass, independent of body fat percentage, that is positively associated with BMD. Indeed, lean body mass has been found to correlate with appendicular ${ }^{27}$ and axial ${ }^{28}$ bone mass in previous studies. Further, muscle strength and lean mass have been reported to be positively associated with bone mass in a variety of weight bearing and non-weight bearing sports. ${ }^{23} 29$ Thus the force exerted by the muscle pull on the skeleton during muscular contraction may be a powerful osteogenic stimulus, ${ }^{30}$ although it has been suggested that, for the oestrogenic stimulus to have an effect on BMD, a regular menstrual cycle may need to be present ${ }^{1231-32}$ However, we found no significant association between menstrual irregularity and BMD at the lumbar spine or femoral neck. Moreover, there was no significant association between oral contraceptive use and BMD at the lumbar spine or femoral neck from 1-192 months of use. This agrees with the findings of Prior $e t a l^{33}$ that use of oral contraceptives did not affect bone density. However, other studies have reported positive $\mathrm{e}^{3435}$ and detrimental ${ }^{28}{ }^{35}$ effects of oral contraceptive use on bone mass and fracture risk. ${ }^{36}$ These negative findings need further rigorous scientific studies because of a number of methodological deficiencies, such as not controlling for confounding variables in the study protocols-for example, smoking and endocrine status.

A major variable shown to influence BMD is age. BMD at the lumbar spine and femoral neck has been reported to peak between the third and fourth decades of life, ${ }^{37}$ the second and third decades of life, ${ }^{1}$ or as early as 18 years. ${ }^{38}{ }^{39}$ The age at which this peak occurs and the factors affecting it are of utmost importance in reducing fracture risk. ${ }^{40}{ }^{41}$ We observed that BMD was not significantly associated with age, with lumbar spine BMD peaking at 28 years, after which it declined with age. However, there was no evidence of an age related peak in femoral neck BMD, with a negative exponential decline apparent across the whole age range. This difference may be due to the lower amount of trabecular bone in the femoral neck (25\%) compared with the lumbar spine (35\%), promoting earlier accretion of bone mineral in the former. ${ }^{42}{ }^{43}$

\section{Conclusion}

We observed a negative association between endurance running distance and $\mathrm{BMD}$, and a positive association between body mass and BMD. This suggests that increasing body mass (independently of body fat percentage) may benefit $\mathrm{BMD}$, and that long distance running may be associated with low BMD. As BMD is an important factor in

\section{Take home message}

It may not be the exercise mode alone that affects BMD but the force applied to the limbs during such exercise and the resulting effects on body composition. Therefore planning of exercise regimens may need to consider the type and intensity of exercise undertaken and the subsequent impact on body composition.

the prediction of fracture risk, recommendations on exercise mode and the resulting effects on body composition may need to be considered. The association between distance run and $\mathrm{BMD}$ is a relatively exciting and novel finding which needs to be further investigated through longitudinal research.

\section{Authors' affiliations}

M Burrows, University of East London, School of Health and Biosciences, Strafford Campus, Romford Road, London E15 4LZ

A M Nevill, S Bird, University of Wolverhampton, School of Sport, Performing Arts, and Leisure, Gorway Road, Walsall, West Midlands WSI 3BD, UK

D Simpson, Department of Nuclear Medicine, Kent and Canterbury Hospital, Canterbury, Kent CT1 3NG, UK

\section{REFERENCES}

1 Snow-Harter CM. Bone health and prevention of osteoporosis in active and athletic women. Clin Sports Med 1994;13:389-404

2 Cumming D. Exercise-associated amenorrhoea, low bone density and oestradiol replacement therapy. Arch Intern Med 1996;156:2193-5.

3 Zanker CL. Bone metabolism in exercise-associated amenorrhoea: the importance of nutrition. Br J Sports Med 1999;33:228.

4 Chilibeck PD, Sale DG, Webber CE. Exercise and bone mineral density. Sports Med 1995;19:103-22

5 Okano H, Mizunuma H, Soda MY, et al. Effects of exercise and amenorrhoea on bone mineral density in teenage runners. J Endocrinol 1995;42:271-6.

6 Heinonen A, Oja P, Kannus $P$, et al. Bone mineral density in female athletes representing sports with different loading characteristics of the skeleton. Bone 1995;17:197-203.

7 Grimston SK, Willows ND, Hanley DA. Mechanical loading regime and its relationship to bone mineral density in children. Med Sci Sports Exerc 1993;25:1203-10.

8 Drinkwater BL. Exercise and bones. Am J Sports Med 1996;24:533-5.

9 Zanker CL, Swaine IL. Responses of bone turnover markers to repeated endurance running in humans under conditions of energy balance or energy restriction. Eur J Appl Physiol 2000;83:434-40.

10 Zanker CL, Swaine IL. Relation between bone turnover, oestradiol, and energy balance in women distance runners. Br J Sports Med 1998;2:167-71.

11 Loucks AB. Effects of exercise training on the menstrual cycle: existence and mechanisms. Med Sci Sports Exerc 1990;22:275-80.

12 West RV. The female athlete. Sports Med 1998;26:63-71.

13 Nevill AM, Holder RL. Modelling health related performance indices. Ann Hum Biol 2000;27:543-59.

14 Box GEP, Cox DR. An analysis of transformations [with discussio]. Journal of the Royal Statistics Society Series B 1964;26:211-52

15 World Health Organisation. Assessment of fracture risk and its application to screening for post-menopausal osteoporosis; Technical Report Series 843. Geneva: WHO, 1994.

16 National Health and Nutrition Examination Survey. National Centre for Health Statistics: Plan and operation of the National Health and Nutrition Examination Survey; vital health statistics 1. Hyattsville: DHHS Publication, 1988-1994

17 Lloyd T, Buchanan JR, Bitzer S, et al. Inter-relationships of diet, athletic activity, menstrual status, and bone density in collegiate females. Am J Clin Nutr 1987; 46:681-4

18 Nguyen T, Sambrook P, Kelly P, et al. Prediction of osteoporotic fractures by postural instability and bone density. J Bone Miner Res 1993;307:1111-15.

19 Loucks AB, Verden M, Heath EM. Low energy availability, not stress of exercise, alters LH pulsatility in exercising women. J Appl Physiol 1998;84:37-46

20 Bennell K, Matheson G, Meeuwisse W, et al. Risk factors for stress fractures. Sports Med 1999;28:91-122.

21 Favus MJ. Primer on the metabolic bone diseases and disorders of mineral metabolism. 2nd ed. New York: Raven Press, 1993.

22 Frost HM. The role of changes in mechanical usage set points in the pathogenesis of osteoporosis. J Bone Miner Res 1992;7:253-61

23 Robinson TL, Snow-Harter CM, Taaffe DR, et al. Gymnasts exhibit higher bone mass than runners, despite similar prevalence of amenorrhoea and oligomenorrhoea. J Bone Miner Res 1995;10:26-32. 
24 Morris FL, Naughton GA, Gibbs JL, et al. Prospective ten-month intervention in premenarcheal girls: positive effects on bone and lean mass. J Bone Miner Res 1997; 12: 1453-62

25 Rubin CT, Lanyon LE. Regulation of bone mass by mechanical strain magnitude. Calcif Tissue Int 1985;37:411-17.

26 Frederick EC, Hagy JL. Factors affecting peak vertical ground reaction forces in running. Int J Sport Biomech 1986;2:41-9.

27 Bauer DC, Brauner WS, Cauley JA, et al. Factors associated with appendicular bone mass in older women. Ann Med 1993;1 18:657-64.

28 Sowers MF, Kshirsagar A, Crutchfield M. Body composition, age and femoral neck bone mass of young adult women. Ann Epidemiol $1991 ; 1: 245-54$

29 Snow-Harter CM, Bousxsein ML, Lewis BT, et al. Effects of resistance and endurance exercise on bone mineral status of young women: a randomised exercise intervention trial. J Bone Miner Res 1992:7:761-9.

30 Wolff J. Das Gestets der Transformation der Knocken. Berlin: A Hirshewold, 1892.

31 Cook SD, Harding AF, Thomas KA. Trabecular bone density and menstrual function in women runners. Am J Sports Med 1987;15:503-7.

32 Drinkwater BL, Nilson $\mathrm{K}$, Chesnut $\mathrm{CH}$, et al. Bone mineral content of amenorrhoeic and eumenorrhoeic athletes. N Engl J Med 1984;311:277-81.

33 Prior JC, Kirkland SA, Joseph L, et al. Oral contraceptive use and bone mineral density in premenopausal women: cross-sectional, population-based data from Canadian Multicenter Osteoporosis Study. Can Med Assoc J 2001;165:1023.

34 Bembem DA, Boileau RA, Bahr JM, et al. Effects of oral contraceptives on hormonal and metabolic responses during exercise. Med Sci Sports Exerc 1992:24:434-41.
35 Lindsay R, Tohme J, Kanders B. The effect of oral contraceptive use on vertebral bone mass in pre and postmenopausal women. Contraception 1986;34:333-40.

36 Hartard $M$, Bottermann P, Bartenstein P. Effects on bone mineral density of low-dosed oral contraceptives compared to and combined with physical activity. Contraception 1997;55:87-90

37 Prior JC, Kreiger JN, Tenehouse A. No positive effect of oral contraceptives on bone density: a population-based cross-sectional study in women aged 25-45 from the Canadian multicentre osteoporosis study. Bone 1998;23:5304-12.

38 Alkel L, Clasey JL, Fehling PC, et al. Contributions of exercise, body composition, and age to bone mineral density in premenopausal women. Med Sci Sports Exerc 1995;27:1477-85.

39 Bass S, Pearce G, Bradney $M$, et al. Exercise before puberty may confer residual benefits in bone density in adulthood: studies in active prepubertal and retired female gymnasts. J Bone Miner Res $1998 ; 13: 500-7$

40 Bradney M, Pearce G, Naughton G, et al. Moderate exercise during growth in prepubertal boys: changes in bone mass, size, volumetric density, and bone strength: a controlled prospective study. J Bone Miner Res 1998;13:1814-21.

41 Bailey DA, Faulkner RA, McKay HA. Growth, physical activity, and bone mineral acquisition. Exerc Sports Sci Rev 1996;24:233-67.

42 Karlsson $M$, Linden C, Karlsson C, et al. Exercise during growth and bone mineral density and fractures in old age. Lancet 2000;355:46972 .

43 Riggs BL, Wahner HW, Dan WL. Differential changes in bone minera density of the appendicular and axial skeleton with aging. J Clin Invest $1981 ; 67: 328-35$

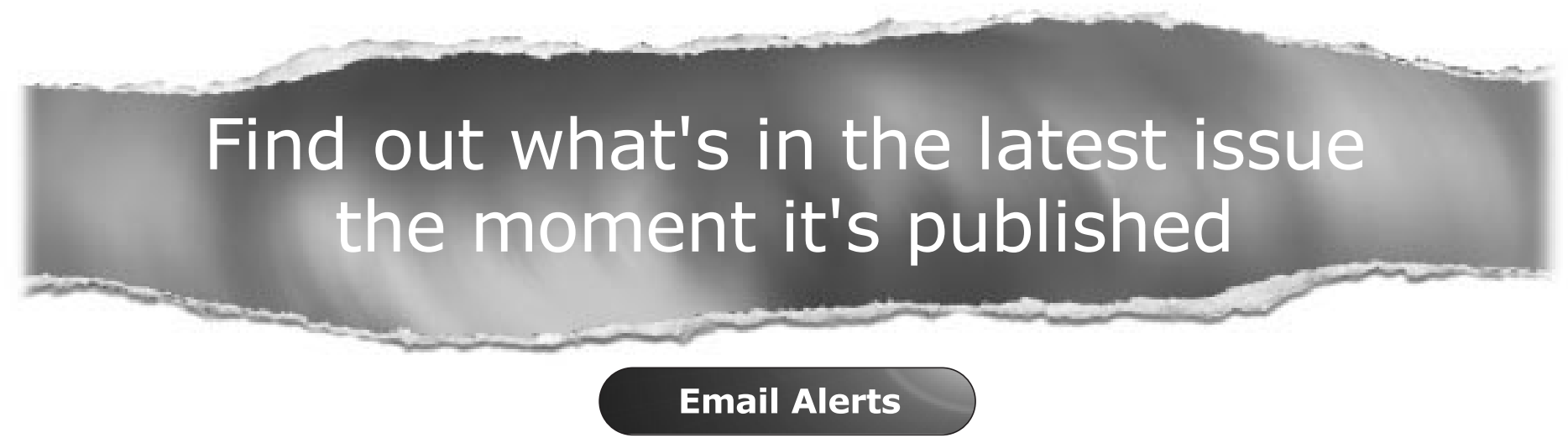

Sign up to receive the table of contents by email every month. You can select from three alerts:

Table of Contents (full), TOC Awareness (notice only); British Journal of Sports Medicine related announcements.

www.bjsportmed.com 